\title{
Rehabilitation of a Patient with Bell's Palsy
}

\author{
Vrushali K. Athawale1, Dushyant P. Bawiskar², Pratik Arun Phansopkar³ \\ 1, 2,3 Department of Musculoskeletal Physiotherapy, Ravi Nair Physiotherapy College, \\ Datta Meghe Institute of Medical Sciences, Wardha, Maharashtra, India.
}

\section{INTRODUCTION}

Facial nerve palsy is the disease of cranial nerve. From the total number of cases, 60 to $75 \%$ of Bell's palsy cases are idiopathic form of facial palsy. Facial nerve palsy results in weakness of facial muscles, atrophy, asymmetry of face and also disturbs the quality of life. Bell's palsy occurs in every class of population affecting people of all the age groups but the most common age group affected is 15 - 50 years with equal sex prediliction accounting $11-40$ cases per 100,000 . If facial palsy is not treated properly then it may result in variety of complications like motor synkinesis, dysarthria, contractures of facial muscles, and crocodile tear. Currently facial paralysis treatment consists of combination of pharmacological therapy, facial neuromuscular re-entrainment physiotherapy or surgical intervention by static and dynamic facial reanimation techniques. Physiotherapy treatment is effective for treating facial paralysis with minimal complications and can be individualized.

Bell's palsy is the idiopathic form of facial nerve palsy which accounts for 60 to 75 $\%$ of cases and male to female ratio is 1:3.1 The aetiology of facial paralysis is not yet thoroughly understood. Cases of varicella-zoster, mononucleosis, herpes simplex virus, mumps and measles have demonstrated good serology in several reports for their association but still stands unclear.2 Peripheral facial nerve palsy may be idiopathic (primary cause) or Bell's palsy (secondary). Causes of the secondary unilateral facial nerve palsy are diabetes, stroke, Hansen's disease, herpes simplex infection, birth injury, trauma, tumour, Guillain-Barre syndrome, and immune system disorders. Causes of the bilateral facial nerve palsy are leukemia, brainstem encephalitis, leprosy, and meningitis. The most prominent current theories of facial nerve paralysis pathophysiology include the reactivation of herpes simplex virus infection (HSV type 1).

Current facial paralysis treatment consists of a combination of pharmacological therapy, facial neuromuscular re-entrainment physiotherapy or surgical intervention by dynamic and static facial reanimation techniques. ${ }^{7}$ This is a diagnosed case of right facial nerve palsy which was treated under physiotherapy department with proper rehabilitation protocol.
Corresponding Author Dr. Pratik Arun Phansopkar, Department of Musculoskeletal Physiotherapy, Ravi Nair Physiotherapy College, Datta Meghe Institute of Medical Sciences, Wardha, Maharashtra, India. E-mail: drpratik77@gmail.com

DOI: $10.14260 / j e m d s / 2021 / 323$

How to Cite This Article: Athawale VK, Bawiskar DP, Phansopkar PA. Rehabilitation of a patient with bell's palsy. J Evolution Med Dent Sci 2021;10(20): 1551-1554, DOI:

10.14260/jemds/2021/323

Submission 08-09-2020,

Peer Review 02-11-2020,

Acceptance 08-11-2020,

Published 17-05-2021.

Copyright (c) 2021 Vrushali K. Athawale et al. This is an open access article distributed under Creative Commons Attribution License [Attribution 4.0 International (CC BY 4.0)] 


\section{PRESENTATION OF CASE}

A 47-year-old male with right hand dominance, farmer by occupation visited the ENT department who was then referred for rehabilitation to physiotherapy department with complaints of slurred pronunciation during speech, lack of movement of right eyebrows, involuntary blinking of right eye, inability to chew the food, wrinkles on forehead, and inabilty to whistle. He had no history of trauma, prolonged exposure of cold winds, or fever. At ENT department several investigations were done like complete blood count, magnetic resonance imaging, and computed tomography scan with proper examination and was diagnosed as Bell's palsy of right facial nerve. All the vitals signs were normal. The laboratory investigations were in normal range with haemoglobin: $14.5 \mathrm{~g}$ / $\mathrm{dL}$, renal function test (serum creatinine $1.3 \mathrm{mg} / \mathrm{dL}$ serum urea: $30 \mathrm{mg} / \mathrm{L}$.

The patient had no significant medical and surgical history. He is a smoker and tobacco chewer but denied alcoholism. The physician prescribed him oral steroids, course of antibiotics along with ophthalmic ointment to prevent corneal abrasions. Patient reported that his facial paralysis came on suddenly and was accompanied with pain in right ear.

The consent form was signed by the patient and examination was done in the sitting position. On inspection there was asymmetry of face, deviation of angle of mouth, inability to close the eye, and drooping of face on right side. On examination, the facial nerve was examined with several movements like blowing air in the mouth, smiling, nasal flaring, elevation of eyebrows, frowning of eyebrows.

The facial nerve provides the motor functions for the facial expression muscles (frontalis, orbicularis oculi, buccinators and orbicularis oris and stapedius, parasympathetic for the tear and submandibular glands and sensory feedback from the two thirds of a tongue's anterior movement. Patients with dry eyes, lowered corneal reflection, drooling, hyperacusis, impaired sensation, otalgia and speech joint disorders can also develop facial droops. This patient usually criticizes all the muscles on one side of the face for weakness or paralysis. The face falls and the fleshy fold go away, the front falls, and the mouth's corner drops. When he attempted to close, his eyelids they wouldn't shut down and the lower eyelids would roll up (bell's phenomenon). Often due to lack of lubrication and excessive contact, the eye becomes irritated, the patient stated.

Tear production decreases; however, due to the loss of control of the lid, tears can appear excessively to be tearing from the eye. Food and saliva will bathe and break from the corner on the affected side of the mouth. A patient whose facial defects are rapidly onset. A history of paralysis initiation and development is significant because it starts slowly over more than two weeks. Medical history of peripheral nerve paralysis includes recent eruption, arthralgia, or fever. The clinical examination involves a screening of the ear canal, tympanic membrane and oropharynx, assessment of the peripheral nerve activity in the limbs of the parotid gland and palpation. Physical research also involves an evaluation of cranial nerve activity and all facial muscles to determine front involvement.

There was difficulty in performing movements. Other cranial nerves were intact. The strength duration curve of patient was taken to know the rate of denervation. The House Brackmann Scale score was 5 (severe dysfunction).

\begin{tabular}{|cc|}
\hline First Encountered Discomfort & $12-06-2020$ \\
\hline Date of ENT Consultation & $13-06-2020$ \\
Date of Physiotherapy Rehab & $14-06-2020$ \\
Date of Initial Examination & $14-06-2020$ \\
Date of Follow-up & $15-07-2020$ \\
\hline Timeline \\
\hline
\end{tabular}

\begin{tabular}{|ccc|}
\hline Sl. No. & Muscles & Grades \\
1 & Frontalis & Poor \\
2 & Corrugator & Fair \\
3 & Orbicularis Oculi & Trace \\
4 & Buccinator & Trace \\
5 & Nasalis & Fair \\
6 & Orbicularis Oris & Fair \\
7 & Mentalis & Poor \\
\hline \multicolumn{3}{|c}{ Table 1. Manual Muscle Grading Pre-Treatment } \\
\hline
\end{tabular}

\begin{tabular}{|ccc|}
\hline Sl. No. & Muscles & Grades \\
1 & Frontalis & Fair \\
2 & Corrugator & Good \\
3 & Orbicularis Oculi & Poor \\
4 & Buccinator & Poor \\
5 & Nasalis & Good \\
6 & Orbicularis Oris & Good \\
7 & Mentalis & Fair \\
\hline \multicolumn{4}{|c}{ Table 2. Manual Muscle Grading Post-Treatment } \\
\hline
\end{tabular}

\section{DISCUSSION OF MANAGEMENT}

\section{Intervention}

Treatment in any facial paralysis rehab includes various strategies to speed up recovery and prevent complications. Four weeks of rehabilitation protocol was carried out with each week adding a new component of treatment. The aim of treatment was to improve facial function.

\section{Week 1:- Phase 1}

Rehabilitation protocol consisted of facial massage. Massage technique was given initially in supine position which includes effleurage, kneading with fingers and thumb, stroking, tapping. Each technique was given for 15 minutes with interval of five minutes. Facial Active exercises were taught to the patient and advised to do at home which is mandatory. The duration of treatment was 2 hours. This protocol was continuing for 1 week.

\section{Week 2:- Phase 2}

On $8^{\text {th }}$ day of rehabilitation, prior to the Electrical Muscle stimulation Superficial heat therapy was given for facial muscles for 15 minutes / session. Electrical Muscle Stimulator (interrupted galvanic current) was initiated with the help of pen electrode in supine position for 45 minutes. The muscles stimulated are frontalis, corrugator, orbicularis oculi, orbicularis oris, buccinator, nasalis, mentalis. Each muscle was stimulated at interval of 2 minutes followed by massage technique. This was continued for the first 2 weeks of rehabilitation.

\section{Week 3:- Phase 3}

The Mirror therapy (active exercises) was initiated, which was performed in front of a mirror in sitting position. The patient was instructed for activities like blowing air in the mouth, nasal flaring, smiling, raising and frowning of the eye brows, opening and closing of the eye, clenching of the teeth for 20 
minutes along with massage and electrical muscle stimulation was also continued in $3^{\text {rd }}$ week of rehab.

\begin{tabular}{|ccc|}
\hline & Pre-Score & Post-Score \\
\hline Resting Symmetry (RS) & 15 & 1 \\
Symmetry of Voluntary Movement & 24 & 56 \\
Synkinesis & 10 & 5 \\
\hline Table 3. Sunnybrook Facial Grading System \\
\hline
\end{tabular}
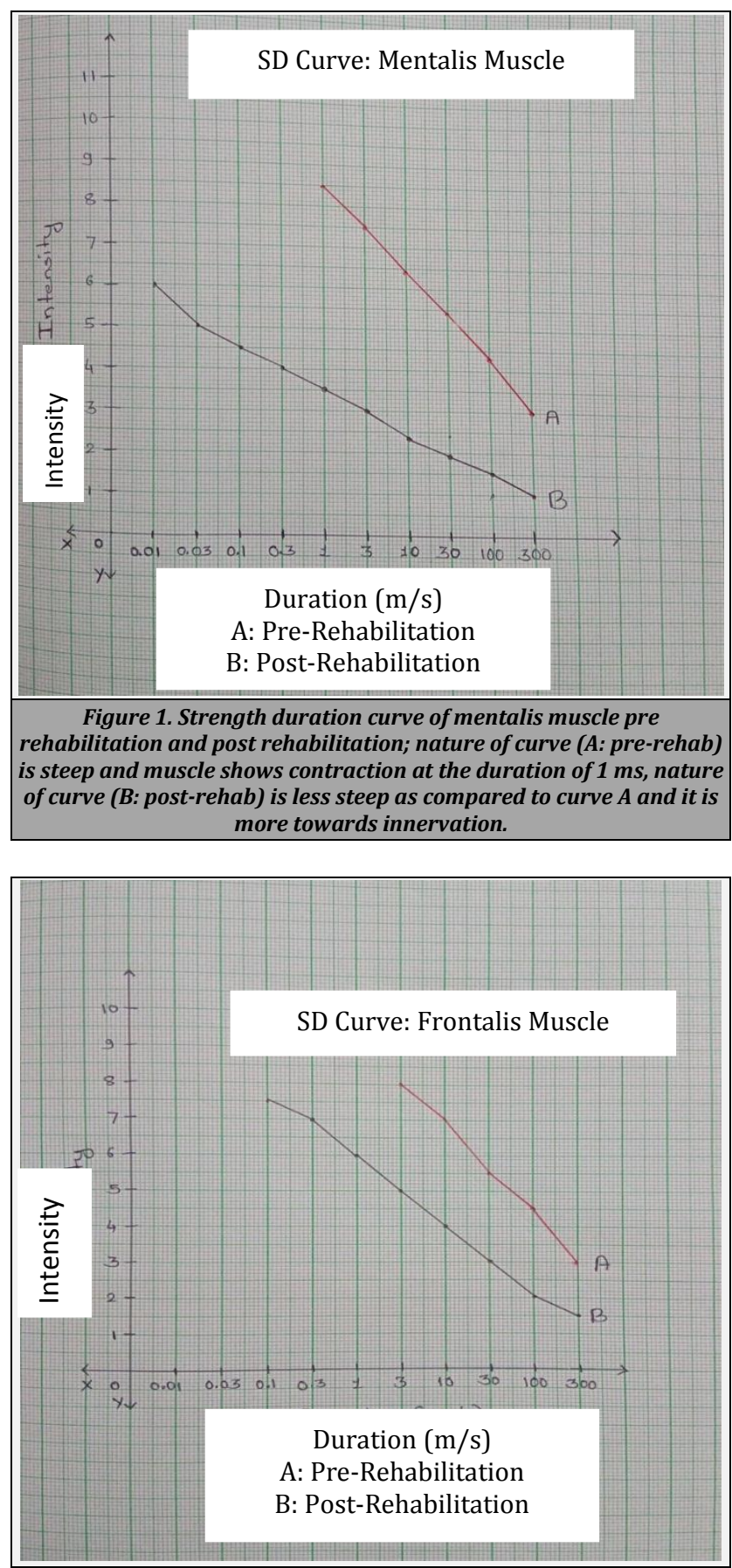

Figure 2. Strength duration curve of frontalis muscle pre rehabilitation and post rehabilitation; nature of curve (A: pre-rehab) is steep and cannot contract to the impulse of shorter duration of less than $3 \mathrm{~ms}$; nature of curve (B: post-rehab) is less steep as compared to pre rehab and more towards innervation.

Week 4:- Phase 4

On $28^{\text {th }}$ day of rehabilitation, resistance training was added in the protocol along with stretching exercises. The patient was instructed to place thumb inside the mouth and stretch the cheek in downward direction for 5 repetitions and maintained the stretch for 30 seconds. The Electrical muscle stimulation was also continued with faradic mode of stimulation for muscle re-education. On $28^{\text {th }}$ day of rehabilitation there were significant improvement in facial functions in muscle strength as described in Table 2, strength duration curve showed status of innervation, House Brackmann Scale Score was 2 (slight dysfunction). Patient was advised to wear sunglasses during the day to prevent from complications.

\section{Outcomes}

On observation the angle of deviation of mouth were reduced, and there were signs of symmetry of face. Patient could blow air on both the side, close the eyes, and could chew properly, and speak. There was improvement in strength duration curve as it is more towards the innervation inclination. The House Brackmann Scale Score was 2. The Sunnybrook facial grading system was evaluated (Table 3 ).

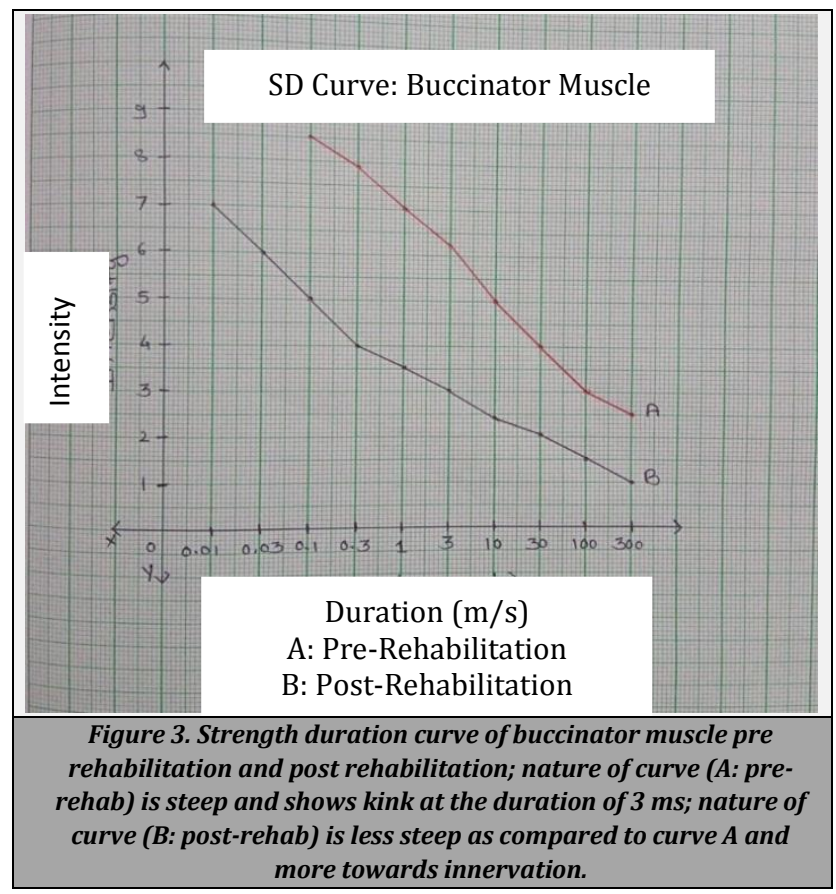

Financial or other competing interests: None.

Disclosure forms provided by the authors are available with the full text of this article at jemds.com.

All authors have contributed substantially for the concept, assessment and evaluation, data acquisition and development of this work. All authors read and approved the final version of the manuscript.

\section{REFERENCES}

[1] Heckmann JG, Urban PP, Pitz S, et al. The diagnosis and treatment of idiopathic facial paresis (Bell's palsy). Dtsch Arztebl Int 2019;116(41):692-702.

[2] Pereira L, Obara K, Dias JM, et al. Facial exercise therapy for facial palsy: systematic review and meta-analysis. Clin Rehabil 2011;25(7):649-58.

[3] Finsterer J. Management of peripheral facial nerve palsy. Eur Arch Otorhinolaryngol 2008;265(7):743-52. 
[4] Pourmomeny AA, Asadi S. Management of synkinesis and asymmetry in facial nerve palsy: a review article. Iran J Otorhinolaryngol 2014;26(77):251-6.

[5] Heidari SF. Recovery in 61-year-old male after bell's palsy: a case report. Annals Journal of Clinical Laboratory Research 2017;5(2):179.

[6] Brach JS, VanSwearingen JM. Physical therapy for facial paralysis: a tailored treatment approach. Phys Ther 1999;79(4):397-404.

[7] Mehta RP. Surgical Treatment of facial paralysis. Clin Exp Otorhinolaryngol 2009;2(1):1-5.

[8] Somasundara D, Sullivan F. Management of Bell's palsy. Aust Prescr 2017;40(3):94-7.

[9] Novak CB. Rehabilitation strategies for facial nerve injuries. Semin Plast Surg 2004;18(1):47-52.
[10] Shafshak TS. The treatment of facial palsy from the point of view of physical and rehabilitation medicine. Eura Medicophys 2006;42(1):41-7.

[11] Goats GC. Massage--the scientific basis of an ancient art: Part 1. The techniques. Br J Sports Med 1994;28(3):14952.

[12] Goldie S, Sandeman J, Cole R, et al. Electrical stimulation treatment for facial palsy after revision pleomorphic adenoma surgery. J Surg Case Rep 2016;2016(4):rjw057.

[13] Bharathi K, Ramya S, Malarvizh D. The Efficacy of mirror therapy in facial palsy among subjects with facial paralysis. International Journal of Research and Scientific Innovation 2019;6(9). 